\title{
Erythropoiesis Stimulating Agents (ESAs) in the Treatment of Cardio-Renal Syndrome Anaemia
}

\author{
Ioannis Koulouridis, Efstathios Koulouridis \\ Department of Nephrology, General Hospital of Corfu, Corfu, Greece \\ Email: koulef@otent.gr
}

Received April 23, 2012; revised June 17, 2012; accepted July 3, 2012

\begin{abstract}
Coexistence of chronic kidney disease (CKD) and chronic heart failure (CHF) define a recently recognized clinical entity known as cardio-renal syndrome. Sufficient evidence suggests that the two pathological conditions share common pathogenic etiology which is not yet fully defined. Superimposed anaemia is a common finding among patients suffering from cardio-renal syndrome. The combination of CKD, CHF and anaemia increase the probability of death by 6 times compared to normal individuals. Early attempts to restore anaemia either by iron supplementation, erythropoiesis stimulating agents (ESAs) or combination of the two have reported to improve quality of life, morbidity and mortality especially among patients treated by cardiologists. Recent publications of well controlled epidemiological studies failed to prove convincing beneficial effect of the above mentioned therapy moreover skepticism has raised concerning the safety of restoring anaemia among patients with cardio-renal syndrome as well as used medications. There are still unresolved problems concerning the definition of anaemia, by means of hemoglobin level among these patients, the target hemoglobin level and the therapeutic regimen of ESAs administration and iron supplementation. We need much more evidence in order to define an effective and safe treatment strategy correcting anaemia among patients with cardio-renal syndrome.
\end{abstract}

Keywords: Cardio-Renal Syndrome; Anaemia; Erythropoiesis Stimulating Agents; Adverse Outcomes

\section{Introduction}

The term cardio-renal syndrome refers to the condition of cardiac and renal failure coexistence when the deterioration of cardiac function implies deterioration of renal function and vice versa. It should not be considered as simple coexistence of two separate pathological conditions but, as a coupled damage of the two organs many of the leading factors are still unknown [1]. According to the consensus conference on cardio-renal syndromes (CRS) held in Venice Italy, 2008 five subtypes of cardiorenal syndromes were recognized [2]. The type 1 or acute cardio-renal syndrome refers to the condition of acute cardiac function worsening which leads to renal injury or dysfunction. The type 2 or chronic cardio-renal syndrome defines the situations of chronic cardiac abnormalities which are coupled with chronic renal dysfunction. The type 3 or acute reno-cardiac syndrome refers to the presence of acute kidney injury which leads to heart injury or dysfunction. The type 4 or chronic reno-cardiac syndrome refers to the presence of chronic kidney disease which leads to chronic cardiac damage or dysfunction and the type 5 or secondary cardio-renal syndrome refers to the presence of systemic pathological conditions affecting simultaneously the two organs such as sepsis, collagen disease, diabetes mellitus, amyloidosis etc. In the present review we shall focus our interest mainly upon the type 2 and type $4 \mathrm{CRS}$ and the effect of anaemia correction upon cardiac and renal function as well as upon patient's final outcome concerning morbidity and mortality.

It is known from the literature that the frequency of kidney disease among patients older than 65 years of age hospitalized for acute decompensated congestive heart failure has been estimated to be $21 \%$ where as $41 \%$ of them exhibit creatinine levels greater than $1.5 \mathrm{mg} / \mathrm{dL}$ [3]. Emergence of acute kidney injury (increment of createnine levels greater than $0.3 \mathrm{mg} / \mathrm{dL}$ ) during therapeutic manipulation of hospitalized patients suffering from congestive heart failure is estimated to be $27 \%-45 \%$ even though they exhibited normal creatinine levels at the admission to the hospital [4]. The presence of cardiac failure among patients suffering from chronic kidney disease (CKD) has been estimated in a large epidemiological survey based upon the data of Medicare system and showed that $39.9 \%$ of patients exhibited cardiac failure from the first visit and an additional 30.7\% developed cardiac failure during the next year [5]. Data from the Acute Decompensated Heart Failure National Registry (ADHERE) study showed that increase of creatinine 
and blood urea nitrogen levels were the most powerful independent risk factors affecting in-hospital mortality [6]. The detailed effect of kidney injury upon mortality of hospitalized and non-hospitalized patients suffering from congestive heart failure was shown from a metaanalysis of sixteen RCTs including 80,018 patients. This study showed a positive correlation between deterioration of renal function and mortality, moreover each 0.5 $\mathrm{mg} / \mathrm{dL}$ increment of creatinine exhibited a $15 \%$ increment of mortality where as for each $10 \mathrm{ml} /$ min reduction of eGFR observed a 7\% increase in mortality [7]. The etiology of increased mortality among patients suffering from cardio-renal syndrome is probably due to increased activity of RAS system, increased sympathetic activity, increased concentration of pro-inflammatory cytokines, deterioration of anaemia, deterioration of left ventricular hypertrophy and myocardial contractility, deranged regulation of extra-cellular volume and final exhaust of the cardiac pump which leads to death [7].

\section{Anaemia in Cardio-Renal Syndrome}

The frequency of anaemia in cardio-renal syndrome is difficult to be estimated because of lack of universal criteria in defining anaemia among patients with cardiac failure. Most authors accept hemoglobin levels lower than $12-12.5 \mathrm{~g} / \mathrm{dl}$ in order to characterize patients with cardiac failure as anemic [8].

Data from the Organized Program to Initiate Lifesaving Treatment in Hospitalized Patients with Heart Failure ((OPTIMIZE-HF) which included 48,612 patients, showed that $51.2 \%$ of them exhibited hemoglobin levels lower than $12.1 \mathrm{~g} / \mathrm{dL}$ whereas $25 \%$ of them exhibited hemoglobin levels between $5 \mathrm{~g} / \mathrm{dL}$ and $10.7 \mathrm{~g} / \mathrm{dL}$. Moreover it was shown that decrease of hemoglobin level by $1 \mathrm{~g} / \mathrm{dL}$ produced an increase in the likelihood of death by $20 \%$ [8].

It is widely accepted that among patients with CKD hemoglobin levels lower than $12 \mathrm{~g} / \mathrm{dL}$ are compatible with anaemia. According to this definition McClellan et al. [9] showed that among 5222 patients with CKD anaemia was present in $47.7 \%$ of them. It was shown also that anaemia was inversely correlated with GFR, so patients with GFR levels greater than $60 \mathrm{ml} / \mathrm{min} / 1.73 \mathrm{~m}^{2}$ exhibited anaemia in a percentage of $26.7 \%$ whereas patients with GFR levels lower than $15 \mathrm{ml} / \mathrm{min} / 1.73 \mathrm{~m}^{2}$ exhibited anaemia in a percentage of $75.5 \%$. The coexistence of cardiac failure, chronic kidney disease and anaemia increases the probability of death by six times compared to healthy individuals [10].

Etiology of anaemia among patients with cardiac failure is attributed to fluid retention and concomitant edema which produce blood dilution as well as to aspirin and antiplatelet drugs which produce chronic occult blood loss. Other causes are reduced absorption of iron, folic acid and vitamin B12 by intestine due to mucosal edema, increased production of inflammatory cytokines such as interleukin 1 or 6 and 18 as well as TNF- $\alpha$ which suppress erythropoiesis and produce cachexia. Another important causative factor of anaemia among these patients is the prescription of angiotensin converting enzyme inhibitors as it is known that Ang-II promotes erythropoiesis in bone marrow. Moreover it is known that converting enzyme is the main catabolic enzyme of the tetra peptide acetyl-N-Ser-Asp-Lys-Pro (AcSDKP) which is a known powerful inhibitor of erythropoiesis so that converting enzyme inhibition leads to increased concentration of AcSDKP with a resultant suppression of effective erythropoiesis [8,10-12].

It has been speculated that patients suffering from cardiorenal syndrome are not deficient of endogenous erythropoietin production. Van der Meer et al. [12] showed in a small number of anemic patients, suffering from cardiac failure and CKD, that $29 \%$ of them exhibited levels of endogenous erythropoietin greater than expected as well as that these patients exhibited greater mortality rate compared to patients with expected or lower than expected levels of endogenous erythropoietin. This finding suggests that a considerable proportion of patients with cardio-renal anaemia exhibit endogenous erythropoietin resistance.

The most important causative factor of anaemia among patients with CKD is the lack of endogenous erythropoietin, absolute or relative, because it is known that about $40 \%$ of patients with CKD exhibit increased levels of bioactive endogenous erythropoietin but reduced response to the hormone action [13]. Other causes of anaemia in CKD patients are the reduced time life of red blood cells, the chronic blood loss from gastrointestinal tract, frequent blood tests, iron deficiency, folate and B12 deficiency, secondary hyperparathyroidism, hypothyroidism, acute or chronic inflammation, aluminum toxicity and hemoglobinopathies [12-14].

\section{Restoration of Cardiorenal Anaemia}

The exogenous administration of erythropoietin and iron, either solely or in combination, is capable to restore anaemia of cardio-renal syndrome and several earlier publications suggest that improve quality of life, left ventricular ejection fraction and morbidity of these patients but newer randomized controlled trials (RCTs) failed to confirm preliminary enthusiasm, on the contrary it is possible that vigorous attempt to anaemia correction increase all cause mortality, cardiovascular mortality and fatal and nonfatal stroke events [15].

The first disappointing results concerning the correction of anaemia in CKD patients came in 2006 published in the same issue of New England Journal of Medicine 
and included the Cardiovascular Risk Reduction by early Treatment with Epoetin beta (CREATE) [16] study and the Correction of Hemoglobin and Outcomes in Renal Insufficiency (CHOIR) [17] study.

The CREATE study included 603 patients with CKD stage III-IV (eGFR: $15-35 \mathrm{ml} / \mathrm{min} / 1.73 \mathrm{~m}^{2}$ ) and mild anaemia (Hb: $11-12.5 \mathrm{~g} / \mathrm{dL})$, patients randomized in two groups, in group I $(\mathrm{n}=301)$ administered erythropoietin (epoetin beta, NeoRecormone, F. Hoffman-La Roche) with target hemoglobin 13.0 - $15 \mathrm{~g} / \mathrm{dL}$, in group II $(\mathrm{n}=$ 302) administered erythropoietin only when hemoglobin levels fell below $10.5 \mathrm{~g} / \mathrm{dL}$ with target hemoglobin lower than $11.5 \mathrm{~g} / \mathrm{dL}$. After a three years observation period no difference in cardiovascular events, deterioration of renal function or left ventricular mass index was noted between groups, in contrast more patients from group I than group II reached end stage renal disease (127 vs $111, \mathrm{p}=$ 0.03 ), general condition and physical activity was improved, as expected, in group I $(p=0.003$ and $p<0.001$ respectively).

The CHOIR study included 1432 patients with CKD stage III-IV (eGFR: $27.0 \pm 8.7 \mathrm{ml} / \mathrm{min} / 1.73 \mathrm{~m}^{2}$ ), patients randomized in two groups, in group I $(n=715)$ administered epoetin alfa with target hemoglobin $13.5 \mathrm{~g} / \mathrm{dL}$ and in group II $(\mathrm{n}=717)$ administered epoetin alfa with target hemoglobin $11.3 \mathrm{~g} / \mathrm{dL}$. After 16 months Data and Safety Monitoring Board discontinued study because of unfavorable outcomes. The end points of the study were all cause of death, myocardial infarction, hospitalization for decompensated heart failure and stoke. A total of 222 events were recorded, 125 of them in group I (high hemoglobin) and 97 in group II (low hemoglobin) [HR = $1.34(95 \%$ CI $1.03-1.74) \mathrm{p}=0.03]$. The recorded events were 65 deaths $(29.3 \%), 101$ hospitalizations for decompensated heart failure (45.5\%), 25 myocardial infarctions $(11.3 \%)$, and 23 strokes $(10.4 \%)$ no difference in the improvement of quality of life was justified between groups.

The above mentioned studies prompted the need to contact double blind placebo controlled trials in order to elucidate the effect of anaemia correction in the evolution of kidney disease and cardiac disease among cardio-renal syndrome patients and so emerged the Study of Anemia in Heart Failure Trial (STAMINA-HeFT) and the Trial to Reduce Cardiovascular Events with Aranesp Therapy (TREAT) study.

The STAMINA-HeFT [18] included 319 patients with symptomatic heart failure $(\mathrm{EF}<40 \%)$ and eGFR $<60$ $\mathrm{ml} / \mathrm{min} / 1.73 \mathrm{~m}^{2}$. Patients randomized in two groups in group I $(\mathrm{n}=157)$ administered placebo in order to keep hemoglobin levels about $11.3 \mathrm{~g} / \mathrm{dL}$ and in group II $(\mathrm{n}=$ 162) administered darbepoetin alfa subcutaneously every two weeks for one year with target hemoglobin $14.0 \pm$ $1.0 \mathrm{~g} / \mathrm{dL}$. No statistically significant difference in exer- cise duration and in heart failure severity according to NYHA criteria was noted between the two groups, although a trend toward a reduction of all cause mortality and hospitalization for acute decompensated heart failure was noted in darbepoetin group.

The TREAT [19] study included 4038 patients with CKD stage III-IV (eGFR: $20-60 \mathrm{ml} / \mathrm{min} / 1.73 \mathrm{~m}^{2}$ ) and diabetes mellitus. Patients randomized in two groups, the darbepoetin alfa group $(n=2012)$ and the placebo group $(n=2026)$. Target hemoglobin for the darbepoetin group was $12 \mathrm{~g} / \mathrm{dL}$ and for the placebo group $9 \mathrm{~g} / \mathrm{dL}$, whenever hemoglobin level fell below $9 \mathrm{~g} / \mathrm{dL}$ in placebo group administered darbepoetin or transfusions but not exceeding hemoglobin level of $11 \mathrm{~g} / \mathrm{dL}$. The end points of the study were all cause mortality, non fatal myocardial infarction, congestive heart failure, stroke, hospitalization for myocardial ischemia and end-stage renal disease (ESRD). Death or cardiovascular events occurred in 632 patients of darbepoetin group and in 602 patients in placebo group $[\mathrm{HR}=1.06(95 \% \mathrm{CI} 0.94-1.17) \mathrm{p}=0.41]$, death or ESRD occurred in 652 patients of darbepoetin group and in 618 patients of the placebo group [HR = $1.06(95 \%$ CI 0.95 - 1.19) $\mathrm{p}=0.29]$. Fatal or nonfatal stroke occurred in 101 patients of the darbepoetin group and in 53 patients of the placebo group [HR $=1.92(95 \%$ CI 1.38 - 2.68) $\mathrm{p}<0.001]$. Blood transfusions needed 297 patients of the darbepoetin group and 496 patients of the placebo group $(p<0.001)$. Only a subtle difference in fatigue was noted among darbepoetin group in relation to the placebo group.

In a later post hoc analysis of the TREAT study Solomon et al. [20] compared the outcomes of participants according to their initial response to darbepoetin. The analysis included 1872 patients from the darbepoetin group authors separated 471 patients who after a two weight-based doses of darbepoetin, in a two-week regimen, failed to increase their hemoglobin levels higher than $2 \%$ of the initial level. These patients characterized as "poor initial responders" and although they received the higher doses of darbepoetin (median dose $232 \mu \mathrm{g}$ vs $167 \mu \mathrm{g}, \mathrm{p}<0.001)$ they exhibited the lower levels of hemoglobin throughout the study. Moreover poor initial responders exhibited greater frequency of cardiovascular events $[\mathrm{HR}=1.31$ (95\% CI 1.09 - 1.59)], greater frequency of all-cause mortality $[\mathrm{HR}=1.41(95 \%$ CI 1.12 $1.72)]$ and greater frequency of stokes $[\mathrm{HR}=1.26(95 \%$ CI 0.78 - 2.02)].

More recently published data indicate that erythropoietin dose rather than hemoglobin level is the cause of inverse outcomes among dialysis and non-dialysis patients. Zhang Y et al. [21] in an observational study included 35,593 elderly patients of Medicare system, undergoing dialysis (19,034 of them were diabetics) the authors showed that the adjusted 9-month mortality risk 
for erythropoietin dose between 15,000 U/week and $45,000 \mathrm{U} /$ week was $13 \%$ among diabetics (95\% CI: 10\% - 16\%) and 5\% among non-diabetics [(95\% CI: 2\% - 9\%) p-value $=0.002]$. Koulouridis I et al. [22] in a Meta-Regression analysis of 31 RCTs included 12,956 dialysis and non-dialysis patients showed that an increase of ESAs dose (erythropoietina equivalent 10,000 U/week increment) was associated with a higher rate of all cause mortality, Incidence Rate Ratio [IRR $=1.42$ (95\% CI: 1.10 - 1.83)] but not cardiovascular mortality, [IRR = 1.09 (95\% CI: 1.02 - 1.18)].

\section{The Holistic Concept of Cardiorenal Patient}

In every day clinical practice a controversy exist between cardiologists and nephrologists concerning the correction of anemia among cardiorenal patients. The controversy is primarily relied upon three unresolved items: Firstly what hemoglobin level characterizes a patient with cardiac failure as anemic, secondly what is the target hemoglobin among these patients, thirdly what is the proper therapeutic regimen (dose and frequency of drug administration).

Reports concerning cardiac failure patients suggest that the ideal hemoglobin levels must be at $13 \mathrm{~g} / \mathrm{dL}$ and preferably between $13.5 \mathrm{~g} / \mathrm{dL}$ and $14 \mathrm{~g} / \mathrm{dL}$ but on the other hand, according to KDOQI guidelines [23], ideal hemoglobin levels for CKD patients must be between 11 $\mathrm{g} / \mathrm{dL}$ and $12 \mathrm{~g} / \mathrm{dL}$ and under no circumstance should they exceed $13 \mathrm{~g} / \mathrm{dL}$ because of an increased hazard concerning side effects due to the increased level of hemoglobin or to erythropoiesis stimulating agents by themselves.

Most of the studies concerning cardiac failure patients are observational studies from various centers with a relatively small number of patients and the validation criteria include improvement of exercise duration, heart failure according to NYHA classification, left ventricular ejection fraction and quality of life which confine the efficacy and not the safety of the drags used. On the other hand it has been proved that among CKD patient's hemoglobin levels greater than $12 \mathrm{~g} / \mathrm{dL}$ are harmful concerning progression of renal disease suggesting that anaemia is a "defense mechanism" which protects glomerulus from further damage and it is possible that these patients are benefited in a narrow limit of hemoglobin level variation [15].

It seems likely that adverse events of anaemia correction are coupled with platelet function and blood viscosity. It has been proven in experimental as well as in clinical studies that hematocrit level greater than $30 \%$ leads to increased platelet aggregation and blood viscosity with deleterious effects upon glomerulus blood supply and acceleration of glomerulosclerosis by a concomitant increase of the likelihood of systemic thromboembolic events. Apart from ESAs the correction of anaemia needs to be administered intravenous iron supplementation and it is known that the transferrin-iron complex is filtered through the glomerulus and in the acidic $\mathrm{pH}$ of urine dissociates to transferrin and divalent iron ions which via the Fenton reaction produce hydroxyl radicals with resultant oxidative stress and tissue damage [15].

The deleterious effect of full anaemia correction is not restricted only upon the kidney, but also encompasses the rest of the cardio-vascular system because increased blood viscosity is coupled with increased peripheral vascular resistance and increased cardiac after-load whereas the decline of renal function increases extracellular volume and blood pressure with resultant congestive heart failure and left ventricular hypertrophy [15].

The uncertainty of ESAs beneficial effect upon cardiorenal anaemia is much more complicated from the observations of van der Meer et al. [12] who investigated 74 anemic patients with congestive heart failure (NYHA class II-IV) and CKD (stage II-III) and found that 29 (39\%) of them exhibited endogenous erythropoietin levels lower than expected, $23(31 \%)$ as expected and 22 $(29 \%)$ higher than expected. Patients with lower levels of endogenous erythropoietin exhibited the lower levels of eGFR which is relevant to reduced erythropoietin production from diseased kidneys on the other hand patients with higher than expected levels of endogenous erythropoietin exhibited increased mortality (log rank: $\mathrm{p}=$ $0.024)$. The multifactorial analysis showed that the ratio $\mathrm{O} / \mathrm{P}$ (Observed/Predicted) of endogenous erythropoietin emerged as the most powerful independent risk factor of increased mortality even if adjusted to variables such as age, gender, hemoglobin levels, NT-pro-BNP levels and eGFR $[H R=1020(95 \%$ CI $1.004-1.036) \mathrm{p}=0.012]$. These results suggest that patients with endogenous erythropoietin resistance exhibit higher mortality rate. On the other hand the results of TREAT study showed that patients with exogenous erythropoietin resistance (nonresponders) exhibited higher mortality rate than the responders. Taken together these findings suggest the possibility that increasing the dose of erythropoietin in any patient who doesn't respond adequately to the hormone action may aggravate the possibility of inverse outcomes. Although it is only a suggestion because endogenous erythropoietin levels did not estimated in TREAT study it is wise to accept Sandhu A.'s et al. [10] opinion that "ESA resistance should not be treated with increase in ESA dose alone".

According to present knowledge there are no convinceing answers to the above mentioned items so it is necessary to conduct prospective controlled trials in order to solve the confliction concerning the beneficial effect of restoring anaemia in patients with cardiorenal syndrome as well as the safety of erythropoietic agents. The 
Reduction of Events with Darbepoetin alfa in Heart Failure (RED-HF) [24] trial and EPOCARES study [25] are now in progress and we are waiting with great interest the results to be published. The eligibility criteria in RED-HF trial concerning anaemia in heart failure patients accepted hemoglobin levels between $9.0 \mathrm{~g} / \mathrm{dL}$ and $12.0 \mathrm{~g} / \mathrm{dL}$, the darbepoetin alfa administered subcutaneously every two weeks until hemoglobin levels reached at $13 \mathrm{~g} / \mathrm{dL}$ and monthly there after, if hemoglobin levels reached $14.5 \mathrm{~g} / \mathrm{dL}$ darbepoetin stopped and placebo was administered [24]. The eligibility criteria in EPOCARES study accepted hemoglobin levels compatible with mild anemia between 10.3 - $12.6 \mathrm{gr} / \mathrm{dL}$ for men and 10.3 $11.9 \mathrm{gr} / \mathrm{dL}$ for women. The study designs encompass three groups of patients with different treatment schedules in order to investigate the hematopoietic and nonhematopoietic effects of erythropoietin [25]. It is obvious that the two studies exhibit considerable discrepancy in anemia definition among CRS patients as well as in target hemoglobin levels.

Meanwhile the Food and Drug Administration (FDA) on June 24, 2011 announced its alert concerning the "Modified dosing recommendations to improve the safe use of Erythropoiesis-Stimulating Agents (ESAs) in chronic kidney disease" according to which stated that: 1) "Using of ESAs to target a hemoglobin level greater than $11 \mathrm{~g} / \mathrm{dL}$ increases the risk of serious adverse cardiovascular events and has not been shown to provide additional patient benefit"; 2) "No clinical trial to date has identified a hemoglobin target level, ESA dose, or dosing strategy that does not increase these risks". Especially for patients with CKD not on dialysis recommends initiating treatment with ESAs when hemoglobin level is less than $10 \mathrm{~g} / \mathrm{dL}$ and if the rate of hemoglobin decline indicates the likelihood of red blood cell transfusion in order to avoid alloimmunization and/or other transfusion-related risks. Finally recommends that: "If the hemoglobin level exceeds $10 \mathrm{~g} / \mathrm{dL}$, reduce or interrupt the dose of ESA and use the lowest dose of ESA sufficient to reduce the need for red blood cell transfusions".

FDA recommendations refers not only to ESRD patients but also to patients with cardio-renal syndrome anaemia especially type 2 and 4 so it is wise to accept these recommendations in clinical practice when we treat with ESAs patients with cardio-renal syndrome anaemia.

In conclusion we have to pay much more attention in treating cardio-renal anaemia either by erythropoiesis stimulating agents or intravenous iron. It seems likely that, according to FDA, the target hemoglobin must not exceed $11 \mathrm{~g} / \mathrm{dL}$. ESAs administration has to begin with the lower recommended doses until target hemoglobin is achieved and then dose titration must be prompted. If patient does not exhibit successful response it is not wise to increase ESAs dose until definite convincing results prove the safety of this strategy.

\section{REFERENCES}

[1] C. Longhini, C. Molino and F. Fabbian Cardiorenal, "Syndrome: Still Not a Defined Entity," Clinical and Experimental Nephrology, Vol. 14, No. 1, 2010, pp. 12-21. doi:10.1007/s10157-009-0257-4

[2] C. Ronco, P. McCollough, S. D. Anker, I. Anand, N. Aspromonte, S. M.Bagshaw, et al., "For the Acute Dialysis Quality Initiative (ADQI) Consensus Group. CardioRenal Syndromes: Report from the Consensus Conference of the Acute Dialysis Quality Initiative," European Heart Journal, Vol. 31, No. 6, 2010, pp. 703-711. doi:10.1093/eurheartj/ehp507

[3] H. M. Krumholtz, Y. T. Chen, V. Vaccarino, Y. Wang, M. J. Radford, W. D. Bradford, et al., "Correlates and Impact on Outcomes of Worsening Renal Function in Patients $\geq$ 65 Years of Age with Heart Failure," American Journal of Cardiology, Vol. 85, No. 9, 2000, pp. 1110-1113. doi:10.1016/S0002-9149(00)00705-0

[4] D. E. Forman, J. Butler, Y. Wang, W. T. Abraham, C. M. O'Conor, S. S. Gottlieb, et al., "Incidence, Predictors at Admission, and Impact of Worsening Renal Function among Patients Hospitalized with Heart Failure," Journal of the American College of Cardiology, Vol. 43, No. 1, 2004, pp. 61-67. doi:10.1016/i.jacc.2003.07.031

[5] R. N. Foley, A. M. Murray, S. Li, C. A. Herzog, A. M. McBean, P. W. Eggers and A. J. Collins, "Chronic Kidney Disease and the Risk for Cardiovascular Disease, Renal Replacement, and Death in the United States Medicare Population, 1998 to 1999," Journal of American Society of Nephrology, Vol. 16, No. 2, 2005, pp. 498-495. doi:10.1681/ASN.2004030203

[6] G. C. Fonarow, K. F. Adams, W. T. Abraham, C. W. Yancy and W. J. Boscardin, "For the ADHERE Scientific Advisory Committee, Study Group, and Investigators. Risk Stratification for In-Hospital Mortality in Acutely Decompensated Heart Failure: Classification and Regression Tree Analysis," The Journal of the American Medical Association, Vol. 293, No. 5, 2005, pp. 572-580.

[7] G. L. Smith, J. H. Lichtman, M. B. Bracken, M. G. Shlipak, C. O. Phillips, P. DiCapua, et al., "Renal Impairment and Outcome in Heart Failure. Systematic Review and Meta-Analysis," Journal of the American College of Cardiology, Vol. 47, No. 10, 2006, pp. 1987-1996. doi:10.1016/j.jacc.2005.11.084

[8] A. Kazory and E. A. Ross, "Anemia: The Point of Convergence or Divergence for Kidney Disease and Heart Failure," Journal of the American College of Cardiology, Vol. 53, No. 8, 2009, pp. 639-647. doi:10.1016/j.jacc.2008.10.046

[9] W. McClellan, S. L. Aronoff, W. K. Bolton, S. Hood, D. L. Lorber, K. L. Tang, et al., "The Prevalence of Anemia in Patients with Chronic Kidney Disease," Current Medical Research and Opinion, Vol. 20, No. 9, 2004, pp. 1501-1510. doi:10.1185/030079904X2763

[10] A. Sandhu, S. Soman, M. Hudson and A. Besarab, "Managing Anemia in Patients with Chronic Heart Fail- 
ure; What Do We Know?" Vascular Health and Risk Management, Vol. 15, No. 6, 2010, pp. 237-252.

[11] D. V. Vlahakos, K. P. Marathias and N. E. Madias, "The Role of the Renin-Angiotensin System in the Regulation of Erythropoiesis," American Journal of Kidney Diseases, Vol. 56, No. 3, 2010, pp. 558-565. doi:10.1053/j.ajkd.2009.12.042

[12] P. van der Meer, D. J. Lok, J. L. Januzzi, P. W. de la Porte, E. Lipsic, J. Van Wijngaarden, et al., "Adequacy of Endogenous Erythropoietin Levels and Mortality in Anaemic Heart Failure Patients," European Heart Journal, Vol. 29, No. 12, 2008, pp. 1510-1515. doi:10.1093/eurheartj/ehn205

[13] J. W. Eschbach and J. W. Adamson, "Anemia of EndStage Renal Disease (ESRD)," Kidney International, Vol. 28, No. 1, 1985, pp. 1-5. doi:10.1038/ki.1985.109

[14] A. K. Abu-Alfa, "CKD Series: Evaluation and Treatment of Anemia in Chronic Kidney Disease," Hospital Physician, Vol. 39, No. 7, 2003, pp. 31-38, 46.

[15] G. Remuzzi and J. R. Ingelfinger, "Correction of AnemiaPayoffs and Problems," The New England Journal of Medicine, Vol. 355, No. 20, 2006, pp. 2144-2146. doi:10.1056/NEJMe068233

[16] T. B. Drueke, F. Locatelli, N. Clyne, K. U. Eckardt, I. C. MacDougall, D. Tsakiris, et al., (for the CREATE Investigators), "Normalization of Hemoglobin Level in Patients with Chronic Kidney Disease and Anemia," The New England Journal of Medicine, Vol. 355, No. 20, 2006, pp. 2071-2084. doi:10.1056/NEJMoa062276

[17] A. K. Singh, L. Szczech, K. L. Tang, H. Barnhart, S. Sapp, M. Wolfson, et al., (for the CHOIR Investigators), "Correction of Anemia with Epoetin Alfa in Chronic Kidney Disease," The New England Journal of Medicine, Vol. 355, No. 20, 2006, pp. 2085-2098. doi:10.1056/NEJMoa065485

[18] J. K. Ghali, I. S. Anand, W. T. Abraham, G. C. Fonarow, B. Greenberg, H. Krum, et al., (for the STAMINA-HeFT Group), "Randomized Double-Blind Trial of Darbepoetin Alfa in Patients with Symptomatic Heart Failure and Anemia," Circulation, Vol. 117, No. 4, 2008, pp. 526-535. doi:10.1161/CIRCULATIONAHA.107.698514
[19] M. A. Pfeffer, E. A. Burdmann, C. Y. Chen, M. E. Cooper, D. de Zeeuw, K. U. Eckardt, et al., (for the TREAT Investigators), "A Trial of Darbepoetin Alfa in Type 2 Diabetes and Chronic Kidney Disease," The New England Journal of Medicine, Vol. 361, No. 21, 2009, pp. 2019-2032. doi:10.1056/NEJMoa0907845

[20] S. D. Solomon, H. Uno, E. F. Lewis, K. U. Eckardt, J. Lin, E. A. Burdmann, et al., (for the TREAT Investigators), "Erythropoietic Response and Outcomes in Kidney Disease and Type 2 Diabetes," The New England Journal of Medicine, Vol. 363, No. 12, 2010, pp. 1146-1155. doi:10.1056/NEJMoa1005109

[21] Y. Zhang, M. Thamer, J. S. Kaufman, D. J. Cotter and M. A. Herman, "High Doses of Epoetin Do Not Lower Mortality and Cardiovascular Risk among Elderly Hemodialysis Patients with Diabetes," Kidney International, Vol. 80, No. 6, 2011, pp. 663-669. doi:10.1038/ki.2011.188

[22] I. Koulouridis, M. Alfayez, T. Trikalinos, E. M. Balk and B. L. Jaber, "Dose of Erythropoiesis-Stimulating Agents (ESAs) and Adverse Outcomes in Chronic Kidney Disease (CKD): A Meta-Regression," Poster Presentation, American Society of Nephrology Kidney Week 2011, 8-13 November 2011, Philadelphia.

[23] National Kidney Foundation KDOQI, “Clinical Practice Guidelines and Clinical Practice Recommendations for Anemia in Chronic Kidney Disease," American Journal of Kidney Diseases, Vol. 47, Suppl. 3, 2006, pp. S11-S15. doi:10.1053/j.ajkd.2006.03.010

[24] J. J. V. McMurray, I. S. Anand, R. Diaz, A. P. Maggioni, C. O'Connor, M. A. Pfeffer, et al., (on Behalf of the RED-HF Committees and Investigators), "Design of the Reduction of Events with Darbepoetin Alfa in Heart Failure (RED-HF): A Phase III, Anaemia Correction, Morbidity-Mortality Trial," European Journal of Heart Failure, Vol. 11, No. 8, 2009, pp. 795-801. doi:10.1093/eurjhf/hfp098

[25] K. Van der Putten, K. E. Jie, M. E. Emans, J. A. Joles, M. J.Cramer, B. K. Velthuis, et al., "Erythropoietin Treatment in Patients with Combined Heart and Renal Failure: Objectives and Design of the EPOCARES Study," Journal of Nephrology, Vol. 23, No. 4, 2010, pp. 363-368. 